\title{
THE
}

\section{The Quenching of Solutions of Some Nonlinear Parabolic Equations}

\author{
Howard A. Levine \\ John T. Montgomery \\ University of Rhode Island
}

Follow this and additional works at: https://digitalcommons.uri.edu/math_facpubs

Terms of Use

All rights reserved under copyright.

\section{Citation/Publisher Attribution}

Levine, H. A., \& Montgomery, J. T. (1980). The Quenching of Solutions of Some Nonlinear Parabolic Equations. SIAM J. Math. Anal., 11(50, 842-847. doi: 10.1137/0511075

Available at: https://doi.org/10.1137/0511075

This Article is brought to you for free and open access by the Mathematics at DigitalCommons@URI. It has been accepted for inclusion in Mathematics Faculty Publications by an authorized administrator of DigitalCommons@URI.For more information, please contact digitalcommons-group@uri.edu. 


\section{IOWA STATE UNIVERSITY \\ Digital Repository}

1980

\section{The Quenching of Solutions of Some Nonlinear Parabolic Equations}

Howard A. Levine

Iowa State University, halevine@iastate.edu

John T. Montgomery

University of Rhode Island

Follow this and additional works at: http://lib.dr.iastate.edu/math_pubs

Part of the Other Applied Mathematics Commons, and the Partial Differential Equations

Commons

The complete bibliographic information for this item can be found at http://lib.dr.iastate.edu/ math_pubs/43. For information on how to cite this item, please visit http://lib.dr.iastate.edu/ howtocite.html.

This Article is brought to you for free and open access by the Mathematics at Iowa State University Digital Repository. It has been accepted for inclusion in Mathematics Publications by an authorized administrator of Iowa State University Digital Repository. For more information, please contact digirep@iastate.edu. 


\title{
THE QUENCHING OF SOLUTIONS OF SOME NONLINEAR PARABOLIC EQUATIONS*
}

\author{
HOWARD A. LEVINE† AND JOHN T. MONTGOMERY
}

\begin{abstract}
We consider the first initial-boundary value problem for $u_{t}=u_{x x}+\phi(u), 0 \leqq x \leqq l$ with $\phi>0$ on $[0, a), \phi$ convex, monotone increasing and $\lim _{u \rightarrow a} \phi(u)=\infty, a<\infty$, and with $u(x, 0) \equiv 0$. If $\Phi(c)=$ $\int_{0}^{c} \phi(\eta) d \eta, \psi(c)=2 \sqrt{2} \int_{0}^{c^{1 / 2}} d y / \phi\left(\Phi^{-1}\left(c-y^{2}\right)\right)$ and $l_{0}=\sup \{\Psi(c) \mid c \in($ Range $\Phi) \cap[0, \infty)\}$, we prove the following: (a) if $l<l_{0}, u$ exists for all $t>0$ and approaches $(t \rightarrow \infty)$, the smallest stationary solution of the differential equation; (b) if $l=l_{0}$ and $l_{0}$ is taken by $\Psi$, then (a) holds; (c) if $l_{0}$ is not taken and Range $\Phi$ is bounded, then $u$ approaches from below the smallest weak stationary solution of the differential equation and this weak solution is not a strong stationary solution, $u_{x x}(l / 2, t) \rightarrow-\infty$, and $u_{t}(l / 2, t) \rightarrow 0$ as $t \rightarrow \infty$; (d) if $l=l_{0}$ and Range $\Phi=[0, \infty)$ or (e) $l>l_{0}$, then the existence interval is finite and $u(l / 2, t) \rightarrow a$ as $t \rightarrow T^{-}$for some $T<\infty$.
\end{abstract}

1. In [3], Kawarada established the following interesting results for the initialboundary value problem:

$$
\begin{aligned}
& u_{t}=u_{x x}+1 /(1-u) \text { for } 0 \leqq x \leqq l, 0 \leqq t<T, \\
& u(0, t)=u(l, t)=0 \text { for } 0 \leqq t<T, \text { and } \\
& u(x, 0)=0 \text { for } 0 \leqq x \leqq l .
\end{aligned}
$$

THEOREM 1. If $l>2 \sqrt{2}$, then $u$ reaches 1 in a finite time along the line $x=l / 2$.

Along with this result, Kawarada was interested in quenching, and proved the following more difficult theorem:

THEOREM 2. If the solution of (1.1)-(1.3) reaches one in finite time, then $u$ is quenched in that time; that is,

$$
\lim _{t \rightarrow T^{-}} \sup _{x}\left|u_{t}(x, t)\right|=\infty \text {. }
$$

Equation (1.1) arises in the study of electric current transients in polarized ionic conductors.

Acker and Walter [1], [2], [5] have considerably sharpened and extended Theorem 1. Among other things, they have shown that for the more general equation $(2.1)$ in the next section, there is a number $l_{0}<\infty$ such that (a) if $l<l_{0}$, the solution exists for all $t \geqq 0$; and (b) if $l>l_{0}$, the solution is defined only on a finite interval $[0, T)$, and $u(l / 2, t) \rightarrow 1^{-}$as $t \rightarrow T$ from below.

The behavior at $l=l_{0}$ was not determined, however, and it is the purpose of this paper to do so. The result appears in Theorem 3 .

This research also duplicates some of the results of Acker and Walter mentioned above. It was done independently before the authors learned of [1], [2], [5]. Additionally, our methods are somewhat different from those of [1], [2], [5].

The techniques we employ allow us to examine, for example, the equation $u_{t}=u_{x x}+(1-u)^{-1 / 2}$ at $l_{0}=4 \sqrt{2} / 3$. This shows that Kawarada's Theorem 2 is not true for the more general case, since although solutions of this equation exist for all time, we have $\sup _{x}|u(x, t)| \rightarrow 1$ as $t \rightarrow \infty$; furthermore, it is $u_{x x}(l / 2, t)$ which blows up, and not $u_{t}(l / 2, t)$, as $t \rightarrow \infty$.

* Received by the editors November 8, 1978 and in revised form November 21, 1979.

$\dagger$ Department of Mathematics, Iowa State University, Ames, Iowa 50011. The work of this author was supported in part by the National Science Foundation under Grant MCS 78-02729.

$\ddagger$ Department of Mathematics, University of Rhode Island, Kingston, Rhode Island 02881 . 
2. The equations are:

$$
\begin{aligned}
& u_{t}=u_{x x}+\phi(u), \\
& u(0, t)=0, \quad u(l, t)=0 \quad \text { for } 0 \leqq t<T, \\
& u(x, 0)=0 \quad \text { for } 0 \leqq x \leqq l,
\end{aligned}
$$

where $\phi$ is continuous on the interval $[0, a)$ and has a continuous positive derivative over this interval, $\lim _{u \rightarrow a^{-}} \phi(u)=\infty$, and $\phi(0)>0$. There is a close relationship between solutions of (2.1)-(2.3) and stationary solutions of (2.1) and (2.2); i.e., solutions of

$$
\begin{aligned}
& 0=f_{x x}(x)+\phi(f(x)) \quad \text { and } \\
& f(0)=f(l)=0 .
\end{aligned}
$$

A weak stationary solution of (2.1) and (2.2) is a once continuously differentiable function $g$ which satisfies (3.1) and (3.2) with the possible exceptions of $x=l / 2$ and

$$
g(x)=\int_{0}^{l} G(x, y) \phi(g(y)) d y,
$$

where $G(x, y)$ is the Green's function associated with the operator $-d^{2} / d x^{2}$ on $[0, l]$ with Dirichlet end conditions at 0 and $l$. That is,

$$
G(x, y) \equiv \begin{cases}\frac{x}{l}(l-y) & \text { for } 0 \leqq x \leqq y \leqq l, \\ \frac{y}{l}(l-x) & \text { for } 0 \leqq y \leqq x \leqq l .\end{cases}
$$

Note that a stationary solution is also a weak stationary solution.

Let us establish the following notation: $\Phi(u) \equiv \int_{0}^{u} \phi(v) d v$ for $0 \leqq u<a$, and $R \equiv\{\Phi(u) \mid 0 \leqq u<a\}$, the range of $\Phi$. Since $\Phi$ is monotone, $\Phi^{-1}$ exists on $R$. We also let $l_{0} \equiv \sup \{\Psi(c) \mid c \in R\}$ where $\Psi(c) \equiv 2 \sqrt{2} \int_{0}^{\alpha}(\Phi(\alpha)-\Phi(\eta))^{-1 / 2} d \eta,(\Phi(\alpha)=c)$. Since $\Phi$ is positive and continuous, $\Psi$ is bounded and $0<l_{0}<\infty$. To see that $l_{0}$ is finite, we make use of the assumptions that $\phi^{\prime}(u)>0$ on $[0, a)$, that $0<\phi(0) \leqq \phi(u)<\infty$ on $[0, a)$, and that $\phi(u) \rightarrow \infty$ as $u \rightarrow a^{-}$. Since $\Phi$ is one to one, $\Psi(c)=\hat{\Psi}(\alpha)$. Thus $l_{0}=\sup \{\hat{\Psi}(\alpha)$; $0 \leqq \alpha<a\}$.

From the mean value theorem, we have two numbers $\eta_{1}, \eta_{2} \in(\eta, \alpha)$ such that

$$
\begin{aligned}
\Phi(\alpha)-\Phi(\eta) & =\Phi^{\prime}(\eta)(\alpha-\eta)+\frac{1}{2} \Phi^{\prime \prime}\left(\eta_{1}\right)(\alpha-\eta)^{2} \\
& =\phi(\eta)(\alpha-\eta)+\frac{1}{2} \phi^{\prime}\left(\eta_{1}\right)(\alpha-\eta)^{2} \\
& \geqq \phi(0)(\alpha-\eta)
\end{aligned}
$$

since $\phi$ is increasing, and

$$
\begin{aligned}
\Phi(\alpha)-\Phi(\eta) & =\Phi^{\prime}(\alpha)(\alpha-\eta)-\frac{1}{2} \Phi^{\prime}\left(\eta_{2}\right)(\alpha-\eta)^{2} \\
& \leqq \phi(\alpha)(\alpha-\eta) .
\end{aligned}
$$

Therefore,

$$
\hat{\Psi}(\alpha) \leqq \sqrt{2} \int_{0}^{\alpha} \frac{d \eta}{\sqrt{\phi(0)(\alpha-\eta)}} \leqq 2 \sqrt{2}(a / \phi(0))^{1 / 2}
$$


while

$$
\hat{\Psi}(\alpha) \geqq \sqrt{2} \int_{0}^{\alpha} \frac{d \eta}{\sqrt{\phi(\alpha)(\alpha-\eta)}}=2 \sqrt{2}(\alpha / \phi(\alpha))^{1 / 2} .
$$

Thus, not only is $l_{0}$ finite but we also have the bounds

$$
\sup _{\alpha \in[0, a)}(\alpha / \phi(\alpha))^{1 / 2} \leqq l_{0} / 2 \sqrt{2} \leqq(a / \phi(0))^{1 / 2} .
$$

We are now ready to state the main result:

THEOREM 3. The number $l_{0}$ is the same as that mentioned in the introduction. Furthermore, if $l=l_{0}$ in (2.1)-(2.3), then exactly one of the following hold:

(a) If there exists $c$ such that $l_{0}=\Psi(c)$, then the solution of (2.1)-(2.3) exists for all $t \geqq 0$. And as $t \rightarrow \infty, u(x, t)$ approaches monotonely from below the smallest stationary solution, which must exist and be bounded away from a.

(b) If $\Psi$ does not attain its supremum, but $\Phi$ has bounded range $R=\left[0, c_{0}\right)$, (i.e., the integral of $\phi$ over $[0, a)$ is $c_{0}, u(x, t)$ exists for all $t$ and approaches monotonely from below the smallest stationary solution $g$, which must be weak but not strong.)

(c) If $l=l_{0}, R=[0, \infty)$, and $\Psi$ does not attain its supremum, then the solution $u(x, t)$ of (2) is defined only in a finite interval $[0, T), u(l / 2, t) \rightarrow$ a as $t \rightarrow T$ from below.

We first state some preliminary results.

LEMMA 1. Let $u(x, t)$ be the solution of $(2.1)-(2.3)$ defined on $[0, l] \times[0, T)$. Then the following hold:

(a) $u$ has continuous derivatives $u_{t t}, u_{t x x}$, and $u_{x x x}$ on $(0, l) \times[0, T)$, and if $T<\infty$ is maximal, we have $\lim _{t \rightarrow T-} u(x, t)=a$ for some $x, 0<x<l^{1}$

(b) $u$ is unique and symmetric about the line $x=l / 2$.

(c) $u_{t}$ is strictly positive when $x \neq 0, x \neq l$.

(d) $u_{x}$ is strictly positive for $0<x<l / 2$, and strictly negative for $l / 2<x<l$. It follows that for each $t, u(\cdot, t)$ is strictly maximized at $x=l / 2$.

For the proof of (b), (c), and (d), see [1]. (a) is a more or less a standard result that follows upon formulating $u$ as a double integral of $\phi$ against Green's function for the heat equation.

The proof of Theorem 3 requires two more preliminary lemmas.

LEMMA 2. The solution $u(x, t)$ of (2.1)-(2.3) exists for all $t \geqq 0$ if and only if there exists a weak stationary solution of $(2.1)$ and $(2.2)$. In this case, $u(\cdot, t)$ approaches uniformly from below the smallest weak stationary solution as $t \rightarrow \infty$.

Proof. Suppose $f$ is a weak stationary solution of (3.1) and (3.2) and $w=f-u$. Then $w$ satisfies at $x \neq l / 2$ (for some $u_{0}$ between $f$ and $u$ ):

$$
\begin{aligned}
& w_{t}=w_{x x}+\phi(f)-\phi(u)=w_{x x}+\phi^{\prime}\left(u_{0}\right) w, \\
& w(x, 0)=f(x), \quad w(0, t)=0, \quad w(l, t)=0, \quad \text { and } \\
& w(l / 2, t) \geqq 0 .
\end{aligned}
$$

It follows from the maximum principle (Theorem 4, p. 173 of [5]) (applied for $x \in(0, l / 2)$, and $x \in(l / 2, l))$ that $w \geqq 0$.

We will first show that $u$ must exist for all $t \geqq 0$ : Since $w_{t}=-u_{t}$ is nonpositive and $\phi(f)-\phi(u)$ is nonnegative, it follows from (5.1) that $w_{x x}(x, t) \leqq 0$ except possibly at $x=l / 2$. However, $w_{x}$ exists and is continuous everywhere; furthermore, it follows from Lemma $1(\mathrm{~d})$ that $w_{x}$ is zero at $x=l / 2$. It is an amusing exercise in elementary calculus to

\footnotetext{
${ }^{1}$ Actually, for our choice of initial values, $x=l / 2$ if $T<\infty$.
} 
show that this implies that $w$ is maximized at $x=l / 2$. If there is a $T$ such that $u$ is defined only for $0 \leqq t<T$, then Lemma 1 implies that $\lim _{t \rightarrow T^{-}} u(l / 2, t)=a$ and the above argument implies that $\lim _{t \rightarrow T^{-}} u(x, t)=f(x)$ uniformly in $x$. We will show this cannot happen unless $T=\infty$. Let $x_{0}<l / 2$. Then on $\left[0, x_{0}\right] \times[0, T], w$ is nonnegative and satisfies $(5.1)$ with $u(x, T) \equiv f(x)$. The maximum principle would then imply that $w(x, T)>0$ for $0<x<x_{0}$. Since $w(x, T) \equiv 0, T=\infty$.

The second step in the proof of this lemma is to show that if $u$ exists for all $t \geqq 0$, then $u$ approaches uniformly from below in a monotone fashion a weak stationary solution $g$.

To see this, let $F(x, t) \equiv \int_{0}^{l} u(y, t) G(x, y) d y$. Then

$$
\begin{aligned}
F_{t}(x, t) & =\int_{0}^{l} u_{t}(y, t) G(x, y) d y \\
& =\int_{0}^{l} u_{x x}(y, t) G(x, y) d y+\int_{0}^{l} G(x, y) \phi(u(y, t)) d y,
\end{aligned}
$$

or

$$
F_{t}(x, t)=-u(x, t)+\int_{0}^{l} G(x, y) \phi(u(y, t)) d y
$$

which is valid on $[0, l]$ for any $t$ for which $u(x, t)<a$. Since $\phi^{\prime}>0$ and $u_{t}>0$, the integrand in (6.2) is monotone in $t$; thus the monotone convergence theorem implies that the right side of (6.2) approaches the limit

$$
J(x) \equiv-g(x)+\int_{0}^{l} G(x, y) \phi(g(y)) d y,
$$

where we have set $g(x)=\lim _{t \rightarrow \infty} u(x, t) \leqq a$. We claim that $J(x)=0$ for all $x$. In view of (6.1) and the fact that $u_{t}>0$, we have that $J \geqq 0$. But if for some $x$, we have $J(x)>0$, then it follows easily that $F(x, t)$ would increase without bound as $t \rightarrow \infty$, and examination of the definition of $F$ reveals that $u$ would reach $a$ in finite time, contrary to assumption. Therefore, $J(x)=0$. Rewriting this, we have that $g$ is a solution of (3.1) and (3.2). It follows that $g$ is continuous, and from (d) of Lemma 1 and the fact that the integral in (4.1) is finite it follows that it is possible that $g(x)=a$ only if $x=l / 2$. Now it is a routine matter to verify that $g$ is continuously differentiable, and at any point $x$ where $g(x) \neq a$, that $g$ is twice differentiable and satisfies (3.1) and (3.2).

LEMMA 3. A weak solution exists if and only if there is a real numberc such that either $c \in R$ and $\psi(c)=l$, or else $c=\int_{0}^{a} \phi(u) d u<\infty$ and $l=\lim _{a \rightarrow c} \psi(d)$. In the latter cases, the weak solution is not strong.

Proof. Let $f$ be a weak solution. It is easy to show that $f$ must be symmetric about $x=l / 2$, and that $f_{x}(l / 2)=0$. On the interval where $f$ satisfies (3.1) and (3.2), $f$ must lie on a level surface of the Hamiltonian "energy" function associated with (3.1); that is,

$$
H\left(f, f_{x}\right) \equiv \frac{1}{2} f_{x}^{2}+\Phi(f)=c,
$$

where $c$ is a constant which, since $f(0)=\Phi(0)=0$ and $f_{x}(l / 2)=0$, must satisfy $c=$ $\frac{1}{2} f_{x}^{2}(0)=\Phi(f(l / 2))$. From (4.1) it follows that $f_{x}(x)$ is positive for $x<l / 2$, so (7) can be rewritten for $0 \leqq x \leqq l / 2$ as

$$
(c-\Phi(f))^{-1 / 2} f_{x}=\sqrt{2},
$$


which can be integrated from 0 to $l / 2$ to get

$$
\int_{0}^{l / 2}(c-\Phi(f(x)))^{-1 / 2} f_{x}(x) d x=l / \sqrt{2} .
$$

Letting $y=f(x)$, we obtain

$$
l=\sqrt{2} \int_{0}^{f(l / 2)}[\Phi(f(l / 2))-\Phi(y)]^{-1 / 2} d y,
$$

and therefore $l=\Psi(c)$.

On the other hand, suppose there is a number $c \in R$ such that (10) holds. Let $f$ be the unique solution of (3.1) with $f(0)=0$ and $f_{x}(0)=(2 c)^{1 / 2}$. Then $f$ is defined for all $x$ such that $f(x)<a$, and $f$ satisfies (7) and therefore (8), as long as $f_{x} \geqq 0$. It is clear from (8) that there must be a point $x_{0}$ such that $f_{x}\left(x_{0}\right)=0$ and $\Phi\left(f\left(x_{0}\right)\right)=c$. Otherwise $f_{x}$ is bounded away from 0 , which would imply that $f$ increases to at least $\Phi^{-1}(c)$, which would in turn imply that $f_{x}$ would decrease to 0 , contrary to assumption. Integrating (8) from 0 to $x_{0}$ yields $\int_{0}^{x_{0}}\left(c-\Phi(f(x))^{-1 / 2} f_{x}(x) d x=\sqrt{2} x_{0}\right.$. Thus,

$$
x_{0}=\frac{1}{2} \sqrt{2} \int_{0}^{f(l / 2)}[\Phi(f(l / 2))-\Phi(y)]^{-1 / 2} d y .
$$

This, with (10), implies that $x_{0}=l / 2$. Since $f$ satisfies $(3.1)$ on $0 \leqq x<l / 2$, and $f(l / 2)=$ $\Phi^{-1}(c)<a, f$ must extend to a strong stationary solution.

Now consider the case where $c=\int_{0}^{a} \phi(u) d u<\infty$, and let $f$ be the unique solution of (8) with $f(0)=0$. Then an argument similar to the previous one shows that $f_{x}(l / 2)=0$, which implies that $f(l / 2)=a$. Defining $f(l / 2+x) \equiv f(l / 2-x)$, we see that $f$ satisfies (7) and is twice differentiable except at $l / 2$, and therefore is a weak stationary solution of (2.1) and (2.2), but not a strong stationary solution.

Example 1 (Kawarada). We examine (1.1)-(1.3), the case where $\phi(u)=(1-u)^{-1}$. Then $\Phi(u)=\int_{0}^{u}(1-r)^{-1} d r=-\ln (1-u), \Phi^{-1}(y)=1-e^{-y}$, and $R=[0, \infty)$. Thus $\Psi(c)=$ $2 \sqrt{2} e^{-b^{2}} \int_{0}^{b} e^{y^{2}} d y$ where $b=\sqrt{c}$. But this is just a multiple of Dawson's integral $D(b)[6]$, whose unique local maximum $D_{0}$ is known to occur at a finite value of $b$. Consequently, if $l<l_{0} \equiv 2 \sqrt{2} D_{0} \approx 1.5303$, there are two equilibrium solutions. Thus, the solution exists for all time. If $l=l_{0}$, then there is one equilibrium solution and still the solution exists for all $t \geqq 0$. Finally, if $l>l_{0}$, there are no equilibrium solutions and $u$ reaches 1 in finite time. This with Theorem 2 implies that quenching occurs in finite time.

Our next example shows that quenching need not occur (in Kawarada's sense) even though $u \rightarrow a$.

Example 2. Examine the case that $\phi(u)=(1-u)^{-1 / 2}$. One can easily compute that $\Phi^{-1}(y)=1-(1-y / 2)^{2}, R=[0,1)$, and $\Phi(\eta)=2\left(1-(1-\eta)^{1 / 2}\right)$. Thus,

$$
\Psi(c)=2 \sqrt{2}\left(c^{1 / 2}-c^{3 / 2} / 3\right),
$$

which is monotone increasing in $(0,1)$. Thus, when $l=l_{0}=4 \sqrt{2} / 3$ we are in case (b) of Theorem 3. In this case, $u \rightarrow 1$, but in infinite time. Furthermore, $u_{t} \rightarrow 0$ so quenching does not occur. When $l<l_{0}$, there is one equilibrium solution and there is no quenching; when $l>l_{0}$, there are no equilibria, and quenching occurs in finite time.

3. We would now like to indicate briefly how the foregoing can help to reveal a more global picture of the semigroup generated by (2.1) and (2.2). There is no reason to assume that $\phi$ is defined only for positive initial data. Indeed, in Kawarada's original equation $\phi$ is defined on $(-\infty, 1)$, and it seems reasonable to ask about nonzero, 
possibly even negative, initial data. Thus, in this section we assume $\phi$ is defined on $(-\infty, a)$, is positive, with $\phi^{\prime} \geqq 0$ and $\lim _{u \rightarrow a^{-}} \phi(u)=\infty$.

THEOREM 4. (a) If (2.1) and (2.2) has no weak stationary solutions, then every solution with continuous initial data reaches a in finite time. (b) If $f$ is a strong equilibrium solution, then any solution with continuous initial data which is everywhere smaller than $f$ exists for all $t \geqq 0$ and remains smaller than $f$.

Proof. Part (b) follows from the maximum principle. To prove (a), let $u$ be a solution of (2.1) and (2.2) with continuous initial data $u_{0}(x)=u(x, 0)$. Then there exists a nonpositive function $v_{0}(x)$ symmetric about $x=l / 2$ with $v_{0}(0)=v_{0}(l)=0, v_{x x}>0$ for $0<x<l$, and $u_{0}(x) \geqq v_{0}(x)$ for all $x$. As before, it follows from the maximum principle's application to the equation $w_{t}=w_{x x}+\phi^{\prime}(v) w$ satisfied by $w=v_{t}$, that if $v(x, t)$ is the solution of (2.1) and (2.2) with $v(x, 0)=v_{0}(x)$, then $v_{t} \geqq 0$ and $v(\cdot, t)$ is symmetric as long as the solution exists. Examination of the proof of Lemma 2 now reveals that if $v$ exists for all $t \geqq 0$, it must increase to a weak stationary solution. Since there are none, it follows that $v$ reaches $a$ in finite time.

\section{REFERENCES}

[1] A. Acker AND W. WAlter, The Quenching Problem for Nonlinear Parabolic Equations, Lecture Notes in Mathematics, 564, Springer-Verlag, 1976.

[2] - On the global existence of solutions of parabolic differential equations with a singular non-linear term, Nonlinear Analysis, 2 (1978), pp. 499-505.

[3] H. KAWARADA, On the solutions of initial boundary problem for $u_{t}=u_{x x}+1 /(1-u)$, Publ. RIMS Kyoto Univ., 10 (1975), pp. 729-736.

[4] M. H. Protter And H. F. Weinberger, Maximum Principles in Differential Equations, Prentice Hall, Englewood Cliffs, NJ, 1967.

[5] W. WALTER, Parabolic differential equations with a singular nonlinear term, Funkcial. Ekvac., 19 (1976), pp. 271-277.

[6] M. Abromowitz And I. A. STEgun, Handbook of Mathematical Functions, Dover Publications, New York, 1965. 